\title{
CIÊNCIA'NATURA
}

\section{Análise espacial dos ventos no Centro de Lançamento de Alcântara, Maranhão}

\author{
Spatial Analysis of winds at the Alcântara Launching Center, Maranhão
}

${ }^{1}$ Vinicius Milanez Couto e ${ }^{2}$ Gilberto Fisch

${ }^{1}$ Instituto Nacional de Pesquisas Espaciais , Brasil

${ }^{2}$ Instituto de Aeronáutica e Espaço, Brasil

\begin{abstract}
Resumo
O vento no Centro de Lançamento de Alcântara (CLA), que apresenta como principais características um comportamento oceânico, com influência dos ventos alísios e da brisa marítima é modificado pela interface oceano-continente e pela topografia local. Por isso, faz-se necessário estudar as suas características do escoamento local, destacando a influência da camada limite superficial e testando a validade da homogeneidade do escoamento em superfície. Neste estudo serão utilizados os dados de duas torres anemométricas instaladas no CLA para avaliar diferenças espaciais nas características do vento médio e de seu ciclo diário. Para fins de comparação, também serão utilizados dados da reanálise ERA5, a quinta geração criada pelo European Centre for Medium-Range Weather Forecasts $(E C M W F)$. Os resultados mostram que as torres anemométricas apresentam, na média, características próximas e que os dados oriundos da reanálise podem ser utilizados para estudos de fenômenos atmosféricos de micro e mesoescala que ocorrem em superfície.
\end{abstract}

Palavras-chave: Torre anemométrica, camada limite superficial, ECMWF ERA5.

\section{Abstract}

The wind at the Alcântara Launch Center (ALC), which presents as main characteristics an oceanic behavior, influenced by the trade winds and the sea breeze, and modified by the ocean-continent interface and local topography. Therefore, it is necessary to study the characteristics of the site flow, highlighting the influence of the surface boundary layer and the validity of the surface flow homogeneity. In this study will be used the data of two anemometric towers installed in the ALC to evaluate spatial differences in the characteristics of the average wind and its daily cycle. For comparison, data from the ERA5 reanalysis, the fifth generation created by European Centre for Medium-Range Weather Forecasts (ECMWF). The results show that the anemometric towers present, on average, similar characteristics and that the data from the reanalysis can be used for studies of mesoscale atmospheric phenomena that occur on the surface.

Keywords: Anemometric tower, surface boundary layer,ECMWF ERA5. 


\section{Introdução}

Localizado no litoral maranhense, o Centro de Lançamento de Alcântara (CLA) (Figura 1a) foi concebido no território brasileiro como local para lançamento de foguetes. Um dos fatores de maior preocupação na parte de segurança de voo são os cálculos de trajetória, que levam em conta o regime do vento, às rajadas de vento e ao cisalhamento do vento próximo a superfície (Palmerio, 2017).

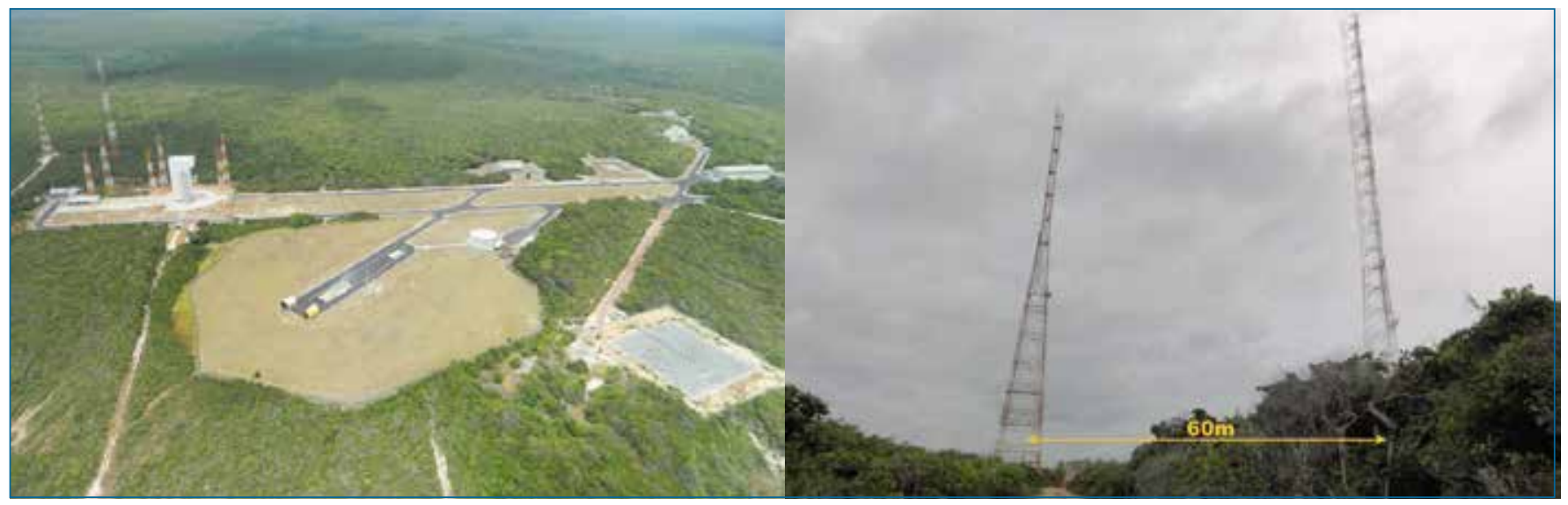

(a)

(b)

Figura 1: CLA: (a) local de lançamento com as torres anemométricas de fundo e (b) disposição das torres.

Em estudos realizados anteriormente, como o de Reuter et al. (2004) e Gisler et al. (2011), indicam que o CLA tem como características ventos de natureza oceânica, persistentes, da direção de nordeste e com velocidade média que varia de 5 a $9 \mathrm{~m} \mathrm{~s}^{-1}$ no decorrer do ano. Isso ocorre principalmente devido ao acoplamento da atuação dos ventos alísios e da circulação de brisa marítima.

Próximo da costa, o CLA possui vegetação nativa e relevo irregular, com elevação que chega a $40 \mathrm{~m}$ e tem como destaque a falésia, que associada aos ventos intensos que sopram do oceano, gera uma camada limite interna, afetando as torres anemométricas e a Torre Móvel de Integração, conforme descrito por Marciotto e Fisch (2013) e Pires et al. (2015).

Magnago et al. (2010), estudando a estabilidade atmosférica no local durante o mês de agosto de 1999, indica que mesmo havendo pequenas diferenças nos valores de estabilidade no decorrer do dia, o vento intenso consegue tornar a turbulência puramente mecânica, com características de quase neutralidade e sem um ciclo diário marcante. Isto também foi recentemente comprovado por Sakagami (2017) para outros sítios experimentais na costa norte do Nordeste do Brasil.

Em um estudo posterior, Medeiros et al. (2013) verifica que há uma certa variabilidade espacial quando se compara dois anemômetros sônicos, um próximo da falésia e outro da torre, sendo a camada limite interna estabilizada em ambos, indicando que a pegada (footprint) na qual são expostos causa essa variabilidade.

Este trabalho tem como intuito contribuir cientificamente nas análises de variabilidade espacial do escoamento do vento no CLA, comparando dados de vento coletados de duas torres anemométricas próximas, procurando observar discrepâncias e verificando se existe validade em considerar o escoamento em superfície homogêneo. Também serão feitas observações do comportamento das reanálises ERA5, recentemente disponibilizado, em relação às torres.

\section{Material e métodos}

Neste trabalho foram utilizados dados no período de 24 de novembro a 15 de dezembro de 2016, com duas torres anemométricas (Figura 1b), uma com 72 m, denominada TA-070, e outra com 100 m, denominada TA-100. Essas torres estão a uma distância aproximada de $60 \mathrm{~m}$ entre si e distantes $600 \mathrm{~m}$ do litoral. Para as análises serão utilizados os conjunto de dados das variáveis velocidade e direção do vento, os quais foram gravados com valores médios a cada 10 minutos de intervalo de tempo.

A TA-070 possui 6 aerovanes da RM Young modelo 05106, montados em espaçamento logarítmico, de 6 a 70 m, enquanto a TA-100 possui 10 anemômetros sônicos da RM Young modelo 86000, espaçados de maneira igual desde 10 até $100 \mathrm{~m}$. Os dados passaram por um controle de qualidade com a remoção dos outliers extremos, representado pelos valores fora da faixa de \pm 3 vezes o desvio padrão centrado na média.

Alguns sensores apresentaram erros sistemáticos significativos para a direção do vento, possivelmente devido ao seu posicionamento. Para estes casos foram utilizadas uma remoção do viés do sensor com o valor médio dos sensores imediatamente acima e abaixo do mesmo. Assumiu-se o fato de que, na camada limite superficial, a direção do vento é constante com a altura. 
Também será utilizado, com finalidade de comparação, as componentes zonal e meridional do vento a 10 e $100 \mathrm{~m}$ do ponto de grade mais próximo dos dados de superfície interpolados para $0,3^{\circ}$ do ERA5 ECMWF (2016), a quinta geração de reanálise e futura sucessora do ERA-Interim, do European Centre for Medium-Range Weather Forecasts (ECMWF). Algumas das novas características adotadas nesta reanálise são um espaçamento de grade de $0,28125^{\circ}(\approx 31,25 \mathrm{~km})$, 137 níveis verticais e saída horária das variáveis meteorológicas.

Para avaliar o vento característico e seu ciclo diário, serão utilizados gráficos de linha, rosas dos ventos e hodógrafos. A comparação os conjuntos de dados serão realizados através da média $(\bar{X})$, mediana $(\tilde{X})$ e desvio padrão $(\sigma)$, além do uso de diagramas de Taylor (Taylor, 2001). Nesse gráfico é apresentado, de forma conjunta, os conjuntos de dados a ser analisado $(f)$ e de referência $(r)$ os valores de raiz da diferença quadrática média centralizada $\left(E^{\prime}\right)$, correlação $(R)$ e $\sigma$, conforme as equações de 1 a 3. Neste trabalho serão utilizados os valores normalizados de $E^{\prime}$ e $\sigma_{f}$, dividindo-se por $\sigma_{r}$. Os diagramas de Taylor em que $f$ são os dados do ERA5, serão computados apenas os valores para horários das torres que coincidam com os horários da reanálise.

$$
\begin{gathered}
R=\frac{\frac{1}{N} \sum_{n=1}^{N}\left[\left(f_{n}-\bar{f}\right)\left(r_{n}-\bar{r}\right)\right]}{\sigma_{f} \sigma_{r}} \\
E^{\prime}=\left\{\frac{1}{N} \sum_{n=1}^{N}\left[\left(f_{n}-\bar{f}\right)-\left(r_{n}-\bar{r}\right)\right]^{2}\right\}^{\frac{1}{2}} \\
\sigma_{f}=\left[\frac{1}{N} \sum_{n=1}^{N}\left(f_{n}-\bar{f}\right)^{2}\right]^{\frac{1}{2}} \quad \sigma_{r}=\left[\frac{1}{N} \sum_{n=1}^{N}\left(r_{n}-\bar{r}\right)^{2}\right]^{\frac{1}{2}}
\end{gathered}
$$

\section{Resultados}

Na Figura 2 é apresentado o hodógrafo da TA-070, TA-100 e do ERA5, com os dados da torre apresentando um padrão diário do vento de nordeste a leste-nordeste. O ERA5 também consegue apresentar um ciclo similar, mas com a direção deslocada em certos horários e com ventos menos intensos.

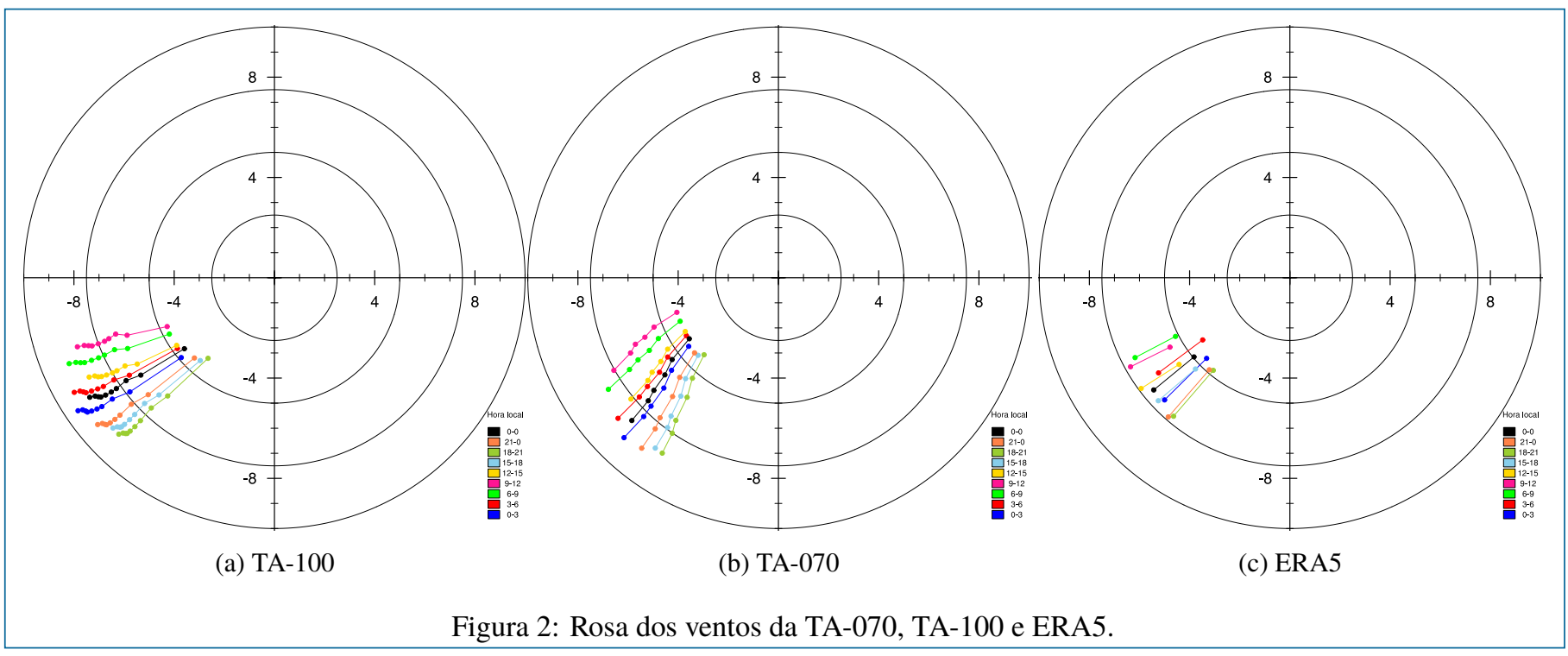

A Rosa dos Ventos na Figura 3 mostra a predominância dos ventos de nordeste, seguido de uma pequena rotação para lestenordeste, com exceção do sensor de $70 \mathrm{~m}$ da TA-100. Acredita-se que esta diferença seja causada por falha na configuração ou montagem do sensor. A reanálise apresenta uma frequência um pouco maior para o vento predominante, com possíveis causas as simplificações da reanálise e a frequência da saída dos dados.

A Figura 4 apresenta o ciclo diário pela decomposição das componentes zonal e meridional do vento para os sensores das torres e para a reanálise. Embora a velocidade do vento da TA-100 possua valores maiores em boa parte dos sensores e que ambas as torres tenham pouca variação no decorrer do dia, ambas as torres apresentam uma forma mais característica ao se observar uma desaceleração na componente zonal, na qual é altamente influenciada pelos ventos alísios, ao mesmo tempo em que se 


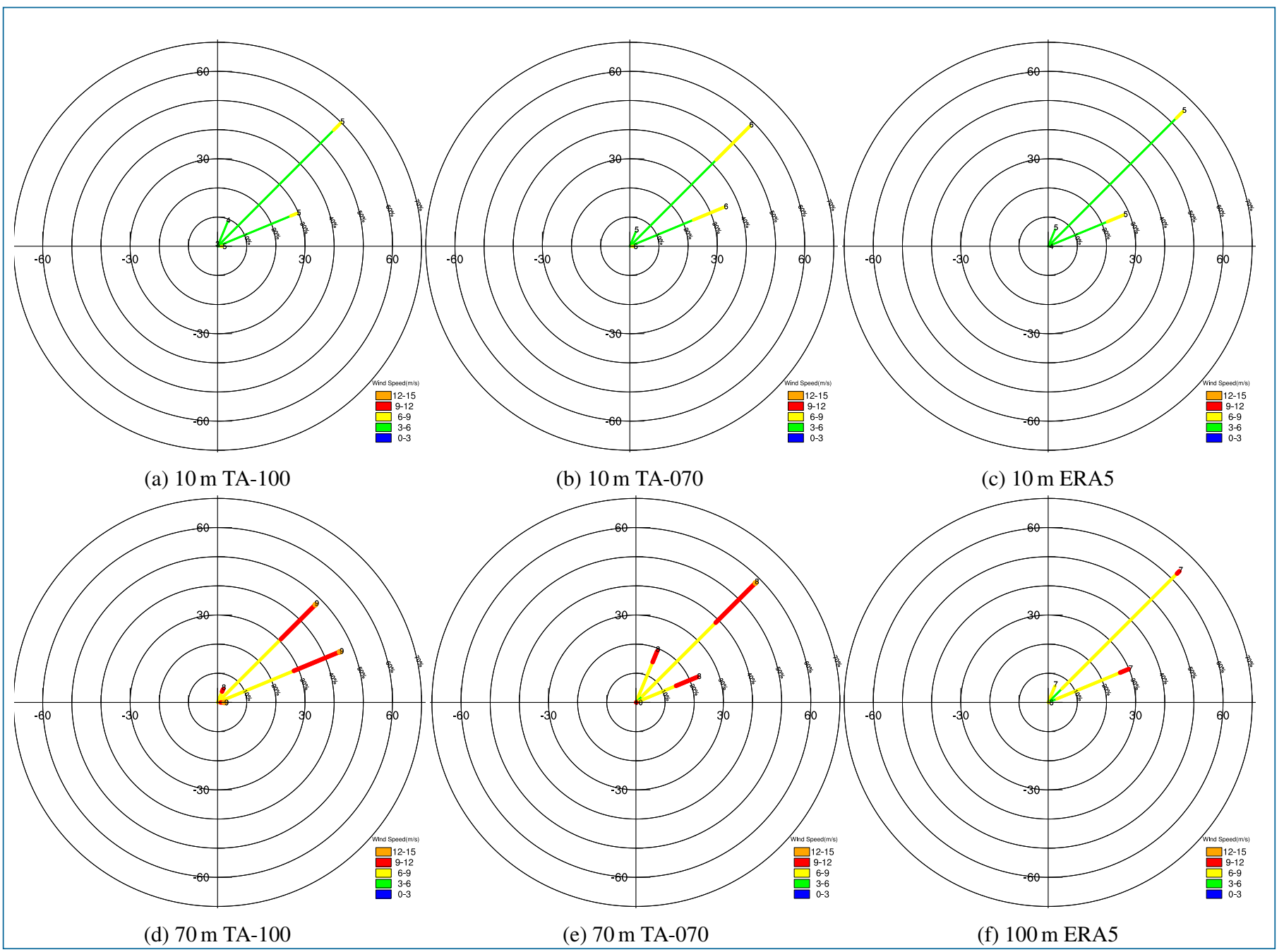

Figura 3: Rosa dos Ventos da TA-070, TA-100 e ERA5 para dois níveis distintos.

intensifica a componente meridional durante o período da tarde, podendo esta ser causada pela circulação de brisa marítima. A diferença na velocidade do vento entre as torres pode ser causada pela proximidade da vegetação no primeiro sensor de cada torre, adicionados pelos diferentes tipos de modelos de anemômetros. No caso dos dados de reanálise, os ventos são mais influenciados pela grande escala, devido o retorno abrupto para a posição mais próxima de leste, sendo que existe uma menor intensificação do vento meridional.

A Tabela 1 também aponta diferença na direção do vento. Percebe-se maiores valores de velocidade do vento no sensor de $10 \mathrm{~m}$ da TA-070 e de $70 \mathrm{~m}$ da TA-100. No entanto, é possível observar valores próximos de $\sigma$ e da diferença entre $\bar{X}$ e $\tilde{X}$. Estes valores estão próximos aos encontrados em estudos como os de Marciotto e Fisch (2013) e Gisler et al. (2011) e indicam também que mesmo com as diferenças encontradas entre cada torre e a reanálise, o ciclo médio consegue ser bem representado, como pode ser visto na Figura 4.

Tabela 1: Média $(\bar{X})$, mediana $(\tilde{X})$ e desvio padrão $(\sigma)$ da TA-070, TA-100 e ERA5.

\begin{tabular}{lcccccccc}
\hline & & \multicolumn{3}{c}{$10 \mathrm{~m}$} & \multicolumn{2}{c}{$70 \mathrm{~m}$} & \multicolumn{2}{c}{$100 \mathrm{~m}$} \\
& & TA-100 & TA-070 & ERA5 & TA-100 & TA-070 & TA-100 & ERA5 \\
\hline \multirow{3}{*}{ Velocidade $\left(\mathrm{m} \mathrm{s}^{-1}\right)$} & $\bar{X}$ & 4,7 & 5,5 & 5,1 & 8,6 & 8,4 & 9,0 & 7,2 \\
& $\tilde{X}$ & 4,6 & 5,5 & 5,1 & 8,6 & 8,4 & 8,9 & 7,1 \\
& $\sigma$ & 0,8 & 0,8 & 0,8 & 1,2 & 1,3 & 1,2 & 1,0 \\
\hline \multirow{2}{*}{ Direção $\left(^{\circ}\right)$} & $\bar{X}$ & 51 & 52 & 50 & 56 & 46 & 57 & 50 \\
& $\tilde{X}$ & 49 & 48 & 49 & 55 & 45 & 57 & 49 \\
& $\sigma$ & 14 & 14 & 11 & 13 & 13 & 13 & 11 \\
\hline
\end{tabular}




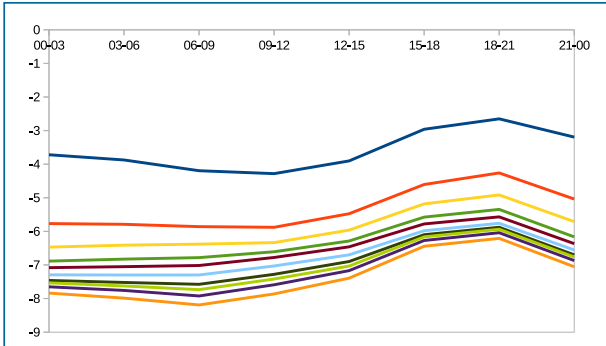

(a) Comp. zonal TA-100

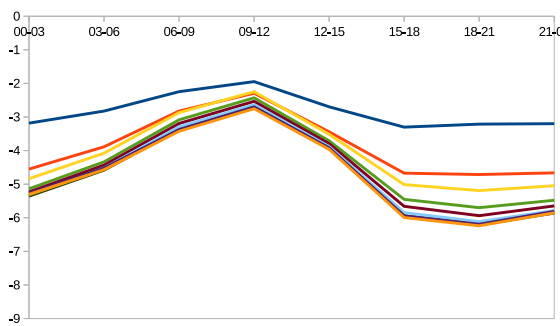

(d) Comp. meridional TA-100

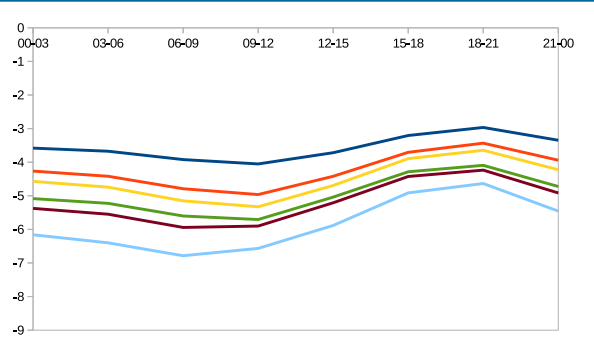

(b) Comp. zonal TA-070

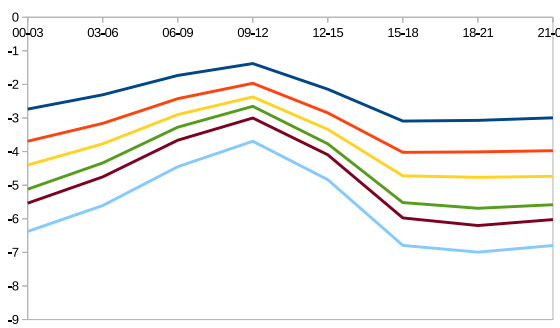

(e) Comp. meridional TA-070

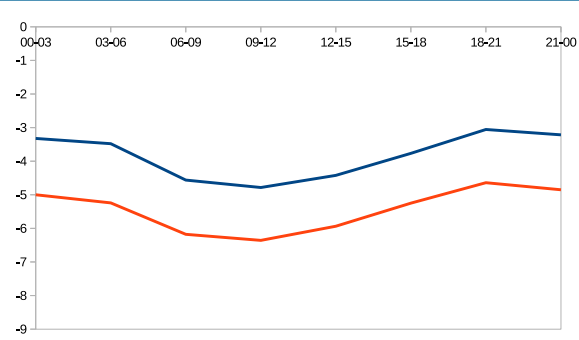

(c) Comp. zonal ERA5

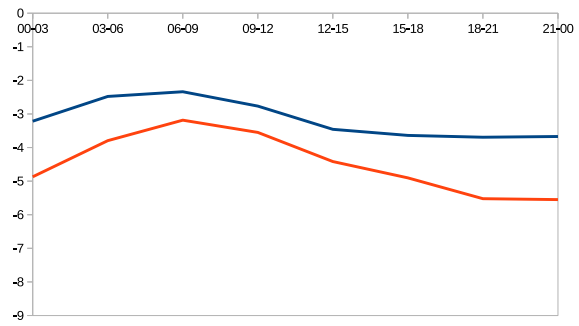

(f) Comp. meridional ERA5

Figura 4: Média de 3 horas das componentes zonal e meridional do vento dos sensores da TA-070 e TA-100 e da reanálise ERA5. Hora Local.

O diagrama de Taylor apresentado na Figura 5a indica que os sensores da TA-070 $(r)$ e a TA-100 $(f)$ apresentam, principalmente para a direção e para as componentes do vento, valores de $R$ acima de $0,8, \sigma_{f} / \sigma_{r}$ próximos de 1,0 e $E^{\prime}$ inferiores a $0,6 \sigma_{r}$, indicando um comportamento semelhante entre as torres anemométricas. Comparando os valores de forma similar a um perfil vertical das componentes do vento, há valores melhores no caso do sensor de $70 \mathrm{~m}$, com destaque para o componente meridional.

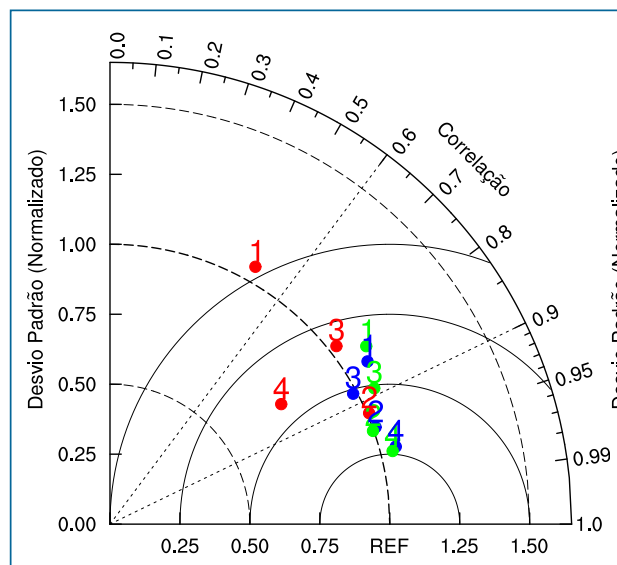

(a) TA-100(f) - TA- $070(r)$

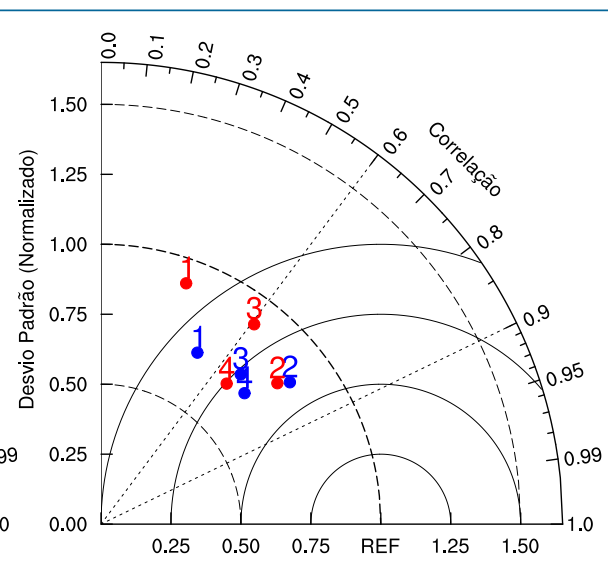

(b) $\operatorname{ERA} 5(f)-\mathrm{TA}-070(r)$

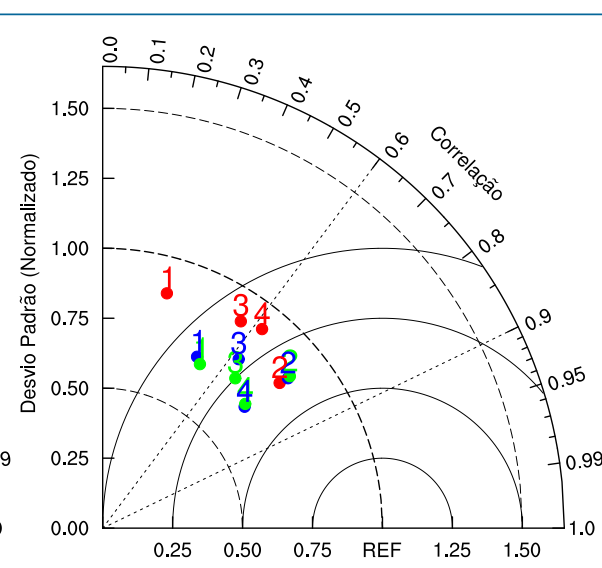

(c) $\operatorname{ERA} 5(f)-\mathrm{TA}-100(r)$

Figura 5: Diagrama de Taylor. $10 \mathrm{~m}$ - vermelho, $70 \mathrm{~m}$ - azul, $100 \mathrm{~m}$ - verde. Variáveis: 1 - velocidade, 2 - direção, 3 - componente zonal, 4 - componente meridional.

Comparando os dados do ERA5 $(f)$ com os sensores das torres ( $r$ ) (Figuras 5b e 5c), apresentam geralmente valores de $R$ superiores a $0,6, \sigma_{f} / \sigma_{r}$ entre 0,7 e 0,9 e $E^{\prime}$ inferiores a $0,8 \sigma_{r}$, sendo esses valores considerados bons, quando se leva em conta a comparação com reanálise. Ao se analisar o perfil vertical das componentes do vento, nota-se que a componente meridional pouco mudou com a altura no caso da TA-070 e uma significativa melhora nos valores no caso da TA-100, enquanto que a componente zonal se comporta de forma similar com a TA-070 e a TA-100.

\section{Conclusões}

Os valores de velocidade e direção do vento apresentados na TA-070 e TA-100 condizem com os estudos anteriores. Levando em consideração os diferentes sensores equipados nas torres anemométricas e a distância entre si, estes presentam valores semelhantes, tanto no seu valor médio como no ciclo diário das variáveis, implicando em uma boa correlação e com valores semelhantes do 
desvio padrão. Pode-se concluir que, na média, o escoamento que passa pelas torres apresentam as mesmas características, o que reforça a hipótese de escoamento horizontal homogêneo.

A reanálise ERA5 apresenta um ciclo diário semelhante aos das torres, sendo mais suavizada e com uma defasagem no tempo para a velocidade e direção do vento, podendo impactar na estatística utilizada. Mesmo assim, apresenta resultado satisfatório. Este conjunto de dados poderá futuramente ser utilizado para estudos de fenômenos atmosféricos de micro e mesoescala, devido a seu espaçamento horizontal e saída horária dos dados.

\section{Referências}

ECMWF (2016). ERA5 reanalysis is in production. ECMWF Newsletter, 147, 7, URL https: //wWw. ecmwf.int/en/ elibrary/16299-newsletter-no147-spring-2016.

Gisler, C. A. F., Fisch, G., de Sousa Correa, C. (2011). Análise estatística do perfil de vento na camada limite superficial no Centro de Lançamento de Alcântara. Journal of Aerospace Technology and Management, 3(2), 193-202, URL http: //dx.doi.org/10.5028/jatm.v3i2.99.

Magnago, R., Fisch, G., Moraes, O. (2010). Análise espectral do vento no Centro de Lançamento de Alcântara (CLA). Revista Brasileira de Meteorologia, 25(2), 260-269, URL http: / / dx. doi .org/10.1590/S0102-77862010000200010.

Marciotto, E. R., Fisch, G. (2013). Investigation of approaching ocean flow and its interaction with land internal boundary layer. American Journal of Environmental Engineering, 3(1), 18-23, URL http: / / article. sapub.org/10 . 5923.j.a jee. 20130301.04. html.

Medeiros, L. E., de Oliveira Magnago, R., Fisch, G., Marciotto, E. R. (2013). Observational study of the surface layer at an ocean-land transition region. Journal of Aerospace Technology and Management, 5(4), 449-458, URL http: / / dx . doi . org/10.5028/jatm.v5i4.244.

Palmerio, A. F. (2017). Introdução à tecnologia de foguetes, $2^{\circ}$ edn. SindCT, São José dos Campos, URL www . s indct . org • br/files/livrofoguetes.pdf.

Pires, L. B. M., Fisch, G., Gielow, R., Souza, L. F., Avelar, A. C., Paula, I. B. D., Girardi, R. D. M. (2015). A study of the internal boundary layer generated at the Alcântara Space Center. American Journal of Environmental Engineering, 5(1A), 52-64, URL article.sapub.org/10.5923.s.ajee.201501.08.html.

Reuter, E. D. D. J., Mota, G. V. D., Fisch, G. F., Cohen, J. C. P. (2004). Estudo observacional da camada limite planetária marinha na região do Centro de Lançamento de Foguetes de Alcântara - MA. Revista Brasileira de Meteorologia, 19(3), 251-264, URL http://www.rbmet.org.br/port/revista/revista_dl.php?id_artigo=73\&id_arquivo=69.

Sakagami, Y. (2017). Influência da turbulência e do perfil de velocidade do vento no desempenho de aerogeradores em dois parques eólicos. Tese de Doutorado, Universidade Federal de Santa Catarina, Florianópolis.

Taylor, K. E. (2001). Summarizing multiple aspects of model performance in a single diagram. Journal of Geophysical Research, 106(D7), 7183 - 7192, URL http://doi.wiley.com/10.1029/2000 JD 900719.

\section{Vinicius Milanez Couto}

Instituto Nacional de Pesquisas Espaciais , Brasil E-mail: vimcless@gmail.com

\section{Gilberto Fisch}

Instituto de Aeronáutica e Espaço, Brasil E-mail:fisch.gilberto@gmail.com 